\title{
Produção de conhecimento sobre as doenças rastreadas pela triagem neonatal no Brasil de 2008 a 2018
}

\author{
Knowledge production about diseases detected by newborn screening in Brazil from 2008 \\ to 2018
}
Producción de conocimiento acerca de las enfermedades detectadas por el tamizaje neonatal en Brasil de 2008 a 2018

Fábio Lima Baggio ${ }^{1 *}$, Nicole Ansbach, Bruna Braga Kapusta, Geisa dos Santos Luz.

\section{RESUMO}

Objetivo: Avaliar a produção de conhecimento acerca da prevalência das doenças triadas pela triagem neonatal no Brasil, entre 2008 a 2018. Métodos: Revisão integrativa na qual a coleta de dados foi realizada nas bases de dados e portais Scientific Electronic Library Online (Scielo), Literatura Latino-Americana e do Caribe em Ciências da Saúde (LILACS) e Biblioteca Virtual em Saúde (BVS). Os dados foram analisados por meio de tabelas e discutidos com a literatura atual e pertinente ao tema. Resultados: No período avaliado foram encontrados 17 estudos. Houve maior produção de conhecimento acerca da prevalência das doenças triadas foi de hemoglobinopatias (doze estudos, incluindo os que tratam apenas de anemia falciforme), seguida por fenilcetonúria (oito estudos) e hipotireoidismo congênito (sete estudos). A menor produção foi sobre deficiência de biotinidase (um estudo). Considerações finais: É evidente que as pesquisas sobre a prevalência das doenças rastreadas pela Triagem Neonatal no Brasil precisam ser ampliadas, pois existe uma discrepância de produção científica ao longo do território nacional. Desse modo, será possível delinear melhores políticas públicas para atender a população-alvo.

Palavras-chave: Triagem neonatal, Prevalência, Perinatologia, Estudos transversais.

\begin{abstract}
Objective: To evaluate the knowledge production about the prevalence of diseases screened by newborn screening in Brazil from 2008 to 2018. Methods: Integrative review in which data collection was performed on databases and portals Scientific Electronic Library Online (Scielo), Latin American and Caribbean Health Sciences Literature (LILACS) and Virtual Health Library (BVS). Data were analyzed through tables and discussed with current and pertinent literature. Results: 17 studies were found. Most of the newborn screening production was about prevalence of hemoglobinopathies (twelve studies including those dealing with sickle cell disease only), followed by phenylketonuria (eight studies) and congenital hypothyroidism (seven studies). The lowest production was about biotinidase deficiency (one study). Final considerations: It is evident that research about prevalence of diseases traced by newborn screening in Brazil need to be expanded, for the reason that there is discrepancy of scientific production throughout national territory. Therefore, it would be possible to plan better public policies to serve the target population.
\end{abstract}

Keywords: Newborn screening, Prevalence, Perinatology, Cross-sectional studies.

\section{RESUMEN}

Objetivo: Evaluar la producción del conocimiento a cerca la prevalencia de las enfermedades detectadas por el tamizaje neonatal en Brasil, entre 2008 y 2018. Métodos: Revisión integradora en la que la recopilación de datos se realizó en las bases de datos y portales Scientific Electronic Library Online (Scielo), Literatura Latinoamericana y del Caribe en Ciencias de la Salud (LILACS) y Biblioteca Virtual de Salud (BVS). Los datos fueron analizados a través de tablas y discutidos con la literatura actual y pertinente. Resultados: Durante el período analizado ha sido encontrado 17 estudios. La mayor parte de la producción del conocimiento a cerca de la prevalencia de las enfermedades detectadas fue de la hemoglobinopatías (doce estudios, incluido los

${ }^{1}$ Centro Universitário de Maringá (UNICESUMAR) Maringá - PR. *E-mail: fabiolimabaggio@yahoo.com.br 
que solo tratan la anemia falciforme), seguida de fenilcetonuria (ocho estudios) e hipotiroidismo congénito (siete estudios). La menor producción fue la de deficiencia de biotinidasa (un estudio). Consideraciones finales: Es evidente que la investigación sobre la prevalencia de enfermedades detectadas por el tamizaje neonatal en Brasil debe ampliarse, ya que existe una discrepancia en la producción científica en todo el territorio nacional. Por lo tanto, será posible planear mejores políticas públicas para servir a la población objetivo.

Palabras clave: Tamizaje neonatal, Prevalencia, Perinatología. Estudios transversales.

\section{INTRODUÇÃO}

A Triagem Neonatal (TN), popularmente conhecida como "Teste do Pezinho", consiste na detecção precoce de patologias na população de recém-nascidos $(R N)$ após as $48 \mathrm{~h}$ de vida, idealmente entre o 3으 e 0 $5^{\circ}$ dia de vida (BRASIL, 2016). A importância dos programas demográficos de Triagem Neonatal é enfatizada e recomendada pela Organização Mundial da Saúde (OMS) desde meados dos anos 1960, a fim de reduzir a incidência de deficiência mental e morbidade em recém-nascidos, especialmente em países em desenvolvimento (LEÃO LL e AGUIAR MJB, 2008).

Uma associação de Pais e Amigos dos Excepcionais (APAE), na cidade de São Paulo em 1976, foi a pioneira em instituir uma tentativa de Triagem Neonatal no Brasil. Essa proposta, a qual realizava apenas a triagem para fenilcetonúria, foi também a dianteira na América Latina (BRASIL, 2002). Uma portaria ministerial do início do século atual (Portaria GM/MS n.을 de 6 de junho de 2001) foi a normativa determinante para a incorporação da Triagem Neonatal ao Sistema Único de Saúde (SUS), que iniciou o Programa Nacional de Triagem Neonatal (PNTN).

O PNTN se tornou a maior iniciativa do SUS na área de genética, com o objetivo de ampliar a TN já existente, detectar patologias precocemente, prevenir e diminuir os impactos e sequelas causadas pelas doenças triadas, além de proporcionar uma busca ativa pelos pacientes suspeitos. Tais propósitos permitem uma produção de dados que possibilitam uma abordagem mais efetiva da situação brasileira (BRASIL, 2016; LEÃO LL e AGUIAR MJB, 2008).

A TN é uma prática que possibilita a detecção precoce de doenças congênitas, caracterizando seu papel como triagem e não diagnóstico das doenças, já que para essas é necessário a realização de exames específicos. A detecção precoce, quando realizada no período oportuno, é realizada enquanto o portador ainda está assintomático, sendo então fundamental para identificação de necessidade de intervenção terapêutica, diminuindo as consequências associadas a cada uma das doenças triadas (NUNES AKC, et al., 2013; COSTA SN, et al., 2012). Algumas das doenças têm significativa taxa de mortalidade, sendo por isso importante a realização do exame (VESPOLI S, et al., 2011).

As doenças triadas pelo PNTN atualmente são hemoglobinopatias (HB), deficiência da biotinidase (DBT), fenilcetonúria (PKU), hipotireoidismo congênito $(\mathrm{HC})$, fibrose cística $(F C)$ e hiperplasia adrenal congênita (HAC) (BRASIL, 2016). Existe também no Brasil o teste do pezinho ampliado, que consta com duas versões que detectam 10 e 50 tipos de doenças, não sendo disponível gratuitamente no SUS (SBP, 2019).

Dentre as hemoglobinopatias, que são causadas por mutações em moléculas da hemoglobina, a anemia falciforme (AF) é a mais frequente. Proveniente do continente africano e introduzida ao Brasil pelo tráfico de escravos, ela é frequente no país devido à miscigenação (BALENSIEFER TK e YAMAGUCHI MU, 2018; REIS FMS, 2018). As manifestações típicas são crises álgicas, síndrome torácica aguda e complicações cardiovasculares, além de maior risco de infecções (BALENSIEFER TK e YAMAGUCHI MU, 2018).

A deficiência de biotinidase e a fenilcetonúria geram deficiência intelectual, sendo que a deficiência de biotinidase pode ocasionar ainda crises epiléticas e dermatites; enquanto a fenilcetonúria pode causar microcefalia (LUZ GS, et al., 2008; BRASIL, 2016). O hipotireoidismo congênito ocasiona retardo mental, atraso de desenvolvimento e hipotonia muscular (LUZ GS, et al., 2008; SOUZA AS, et al., 2018).

A fibrose cística resulta em disfunção respiratória, lesão pulmonar, infecções respiratórias reincidentes e baixo desenvolvimento estrutural (BRASIL, 2016; BONFIM IM, et al., 2019; LUZ GS, et al., 2008). A hiperplasia 
adrenal congênita gera insuficiência de glicocorticoides, mineralocorticoides e excesso ou falta de hormônios andrógenos, com perda de sal e virilização (BRASIL, 2016; WITCHEL SF, 2019; KOPACEK C, et al., 2019).

Não existem dados oficiais públicos pelo Ministério da Saúde (MS) que mostre frequência dessas doenças na população brasileira, o que dificulta a compreensão do cenário das doenças triadas. Dessa forma, a formulação de políticas públicas efetivas às necessidades dessa população é inviabilizada. Neste contexto, objetiva-se avaliar a produção de conhecimento acerca da prevalência das doenças rastreadas pela triagem neonatal, no período de 2008 a 2018 no Brasil.

\section{MÉTODOS}

Trata-se de uma revisão integrativa da literatura para alcance do objetivo proposto. Este estudo possibilita a síntese do conhecimento do assunto, apontando lacunas que precisam ser preenchidas com a realização de novos estudos, como suporte à tomada de decisão e à melhoria da prática clínica, além de permitir a realização de uma síntese de múltiplos estudos publicados, viabilizando conclusões gerais a respeito da TN no Brasil (BENEFIELD LE, 2003).

Na operacionalização dessa revisão, foram percorridas as seguintes etapas: delimitação da questão de pesquisa; estabelecimento dos critérios de inclusão/exclusão para a seleção dos estudos a serem analisados; definição das informações a serem extraídas dos estudos selecionados; avaliação dos estudos incluídos na revisão integrativa; análise dos dados, interpretação dos resultados e apresentação da síntese da revisão (MENDES KDS, et al., 2008).

Com relação às hemoglobinopatias, os subtipos ( $\mathrm{HbS}, \mathrm{HbC}, \mathrm{HbA}$, etc.) foram agrupados como "hemoglobinopatias". Estudos que trataram especificamente de anemia falciforme foram identificados na análise deste estudo como "anemia falciforme".

O levantamento bibliográfico foi realizado pela Internet, por meio da Biblioteca Virtual em Saúde (BVS) e nas bases de dados Literatura Latino-Americana e do Caribe em Ciências da Saúde (LILACS) e Scientific Electronic Library Online (SciELO).

Para o levantamento dos estudos, utilizou-se os seguintes descritores: "prevalência", "triagem neonatal" e "perinatologia", combinado aos termos "fibrose cística", hemoglobinopatias", "hipotireoidismo congênito", "deficiência de biotinidase", "fenilcetonúria" e "hiperplasia adrenal congênita" utilizados para o refinamento da amostra.

Os critérios utilizados para a seleção da amostra foram: estudos originais que abordam a prevalência das doenças triadas pelo PNTN no Brasil, escritos na língua portuguesa, abrangendo as diferentes áreas do conhecimento, publicados entre os anos de 2008 e 2018, em periódicos indexados nas bases de dados SCIELO, LILACS e BVS com texto completo disponibilizado on-line.

Os critérios utilizados para exclusão de amostra foram teses (de mestrado, doutorado, residência médica), trabalhos de conclusão de curso não publicados, estudos escritos em outras línguas e estudos não publicados entre 2008 e 2018.

\section{RESULTADOS}

No período avaliado, identificou-se 17 estudos que trouxeram prevalência das doenças triadas pelo PNTN no Brasil. Observou-se que houve uma produção maior entre 2008 a 2010 e um pico de produção entre 2017 e 2018. Na análise, as hemoglobinopatias tiveram preponderância (doze estudos). Outras doenças também tiveram destaque, como fenilcetonúria (oito estudos) e hipotireoidismo congênito (sete estudos). O periódico com mais publicações sobre a temática foi Arquivos Brasileiros de Endocrinologia e Metabologia.

Em relação ao tipo de estudo, obteve-se: dois transversais, um seccional de prevalência, um retrospectivo, um descritivo ambispectivo, três descritivos transversais, um retrospectivo transversal e descritivo, um quantitativo de prevalência, dois descritivos observacionais e transversais, um retrospectivo transversal, um observacional transversal e um descritivo (Quadro 1). 
Revista Eletrônica Acervo Saúde / Electronic Journal Collection Health ｜ ISSN 2178-2091

Quadro 1 - Análise dos estudos selecionados quanto ao autor, doença, tipo de estudo, objetivo e periódico de publicação.

\begin{tabular}{|c|c|c|c|c|}
\hline Autores & Doença & Tipo de estudo & Objetivo & Periódico \\
\hline $\begin{array}{l}\text { CARVALHO DCSN, } \\
\text { et al., } 2017 .\end{array}$ & $\mathrm{PKU}, \mathrm{HC}$ & Pesquisa transversal & $\begin{array}{l}\text { Avaliar a eficácia de um programa de TN em hospital de referência } \\
\text { do Ceará ao longo de } 11 \text { anos. }\end{array}$ & ABCS Health Sci \\
\hline DINIZ D, et al., 2009. & AF & $\begin{array}{l}\text { Estudo seccional de } \\
\text { prevalência }\end{array}$ & $\begin{array}{l}\text { Apresentar a prevalência do traço e da anemia falciforme em } \\
\text { recém-nascidos do DF de } 2004 \text { a } 2006 \text {. }\end{array}$ & Cad. Saúde Pública \\
\hline $\begin{array}{l}\text { NUNES AKC, et al., } \\
2013 .\end{array}$ & $\begin{array}{l}\text { PKU, HC, } \\
F C, H B, H A C\end{array}$ & Pesquisa retrospectiva & $\begin{array}{l}\text { Avaliar a prevalência das patologias } \mathrm{PKU}, \mathrm{HC}, \mathrm{FC}, \mathrm{HB} \text { e HAC no } \\
\text { Estado de Santa Catarina, a fim de delinear o perfil da população } \\
\text { catarinense em relação à essas patologias. }\end{array}$ & $\begin{array}{l}\text { Arq Bras Endocrinol } \\
\text { Metab. }\end{array}$ \\
\hline $\begin{array}{l}\text { SOUZA RAV, et al., } \\
2009 .\end{array}$ & $\mathrm{HB}$ & Descritivo ambispectivo & $\begin{array}{l}\text { Avaliar o programa de triagem neonatal para hemoglobinopatias no } \\
\text { município de Dourados - MS. }\end{array}$ & $\begin{array}{l}\text { Rev. Bras. Hematol. } \\
\text { Hemoter. }\end{array}$ \\
\hline $\begin{array}{l}\text { LOPES FM, et al., } \\
2010 .\end{array}$ & PKU & Descritivo transversal & $\begin{array}{l}\text { Estudar a prevalência da fenilcetonúria em neonatos triados pelo } \\
\text { teste do pezinho no estado de Goiás, bem como verificar a } \\
\text { incidência e a localização dos indivíduos portadores de } \\
\text { fenilcetonúria. }\end{array}$ & $\begin{array}{l}\text { Ensaio e Ciência: } \\
\text { Ciências Biológicas, } \\
\text { Agrárias e da Saúde }\end{array}$ \\
\hline LUZ GS, et al., 2008. & $\begin{array}{l}\mathrm{PKU}, \mathrm{HC} \\
\mathrm{HB}, \mathrm{FC}, \mathrm{DBT}\end{array}$ & Descritivo transversal & $\begin{array}{l}\text { Verificar a prevalência das doenças diagnosticadas pelo PNTN no } \\
\text { município de Maringá, no período de } 2001 \text { a } 2006 \text {. }\end{array}$ & Rev Gaúcha Enferm. \\
\hline $\begin{array}{l}\text { HOLSBACH DR, et } \\
\text { al., } 2008 .\end{array}$ & $\mathrm{HB}$ & $\begin{array}{l}\text { Retrospectivo, transversal } \\
\text { e descritivo }\end{array}$ & $\begin{array}{l}\text { Caracterizar a ocorrência de HbS por genótipos, sexo, idade no } \\
\text { momento do diagnóstico, índice de cobertura e prevalência em } \\
\text { Mato Grosso do Sul no período de } 2000 \text { a } 2005 \text {. }\end{array}$ & $\begin{array}{l}\text { J Bras Patol Med } \\
\text { Lab }\end{array}$ \\
\hline $\begin{array}{l}\text { SIQUEIRA BR, et al., } \\
2009 .\end{array}$ & $\mathrm{AF}$ & $\begin{array}{l}\text { Pesquisa retrospectiva de } \\
\text { coleta de dados }\end{array}$ & $\begin{array}{l}\text { Avaliar a incidência de homozigotos e heterozigotos para HbS e } \\
\text { caracterização do perfil hemoglobínico das formas de anemia } \\
\text { falciforme e traço falcêmico, nos RN que participaram do PNTN no } \\
\text { Instituto Nativida, no estado de Rondônia no ano de } 2003 \text {. }\end{array}$ & Saber Científico \\
\hline
\end{tabular}

REAS/EJCH | Vol.Sup.n.45 | e2596 | DOI: https://doi.org/10.25248/reas.e2596.2020 Página 4 de 12 


\section{Revista Eletrônica Acervo Saúde / Electronic Journal Collection Health ISSN 2178-2091}

\begin{tabular}{|c|c|c|c|c|}
\hline $\begin{array}{l}\text { BALENSIEFER TK e } \\
\text { YAMAGUCHI MU, } \\
2018 .\end{array}$ & $\mathrm{HB}$ & $\begin{array}{l}\text { Estudo quantitativo de } \\
\text { prevalência }\end{array}$ & $\begin{array}{l}\text { Determinar a prevalência de hemoglobinopatias a partir de } \\
\text { resultados de TN arquivados em um laboratório de Maringá, PR. }\end{array}$ & RBAC \\
\hline $\begin{array}{l}\text { REIS FMS, et al., } \\
2018 .\end{array}$ & $\mathrm{HB}$ & $\begin{array}{l}\text { Descritivo, observacional e } \\
\text { transversal }\end{array}$ & $\begin{array}{l}\text { Avaliar a incidência de hemoglobinas variantes nas diferentes } \\
\text { regiões do Estado do Piauí. }\end{array}$ & Einstein (São Paulo) \\
\hline $\begin{array}{l}\text { ELLER R e SILVA } \\
\text { DB, } 2016 .\end{array}$ & AF & $\begin{array}{l}\text { Estudo descritivo, } \\
\text { observacional e transversal }\end{array}$ & $\begin{array}{l}\text { Avaliar o Programa de Triagem Neonatal da Secretaria de Saúde } \\
\text { do Estado de Santa Catarina (PTN-SES/SC) para doença } \\
\text { falciforme de janeiro de } 2003 \text { a dezembro de 2012, em relação à } \\
\text { sua cobertura e à frequência da doença. }\end{array}$ & J Pediatr \\
\hline $\begin{array}{l}\text { BARRA CB, et al., } \\
2012 .\end{array}$ & $\mathrm{HAC}$ & Retrospectivo transversal & $\begin{array}{l}\text { A eficácia da triagem neonatal para redução de morbimortalidade } \\
\text { das crianças com HAC é a principal justificativa para sua } \\
\text { implantação. }\end{array}$ & Rev Assoc Med Bras \\
\hline $\begin{array}{l}\text { MENDES LC, et al., } \\
2013 .\end{array}$ & $\mathrm{PKU}, \mathrm{HC}$ & $\begin{array}{l}\text { Estudo descritivo } \\
\text { transversal }\end{array}$ & $\begin{array}{l}\text { Avaliar o Programa de Triagem Neonatal do Estado do Tocantins } \\
\text { de } 1995 \text { a } 2011 .\end{array}$ & $\begin{array}{l}\text { Arq Bras Endocrinol } \\
\text { Metab }\end{array}$ \\
\hline $\begin{array}{l}\text { STRANIERI I e } \\
\text { TAKANO OA, } 2009 .\end{array}$ & $\mathrm{PKU}, \mathrm{HC}$ & Estudo transversal & $\begin{array}{l}\text { Avaliar o Serviço de Referência em Triagem Neonatal para } \\
\text { hipotireoidismo congênito e fenilcetonúria no MT. }\end{array}$ & $\begin{array}{l}\text { Arq Bras Endocrinol } \\
\text { Metab }\end{array}$ \\
\hline $\begin{array}{l}\text { AMORIM T, et al., } \\
2010 .\end{array}$ & AF & $\begin{array}{l}\text { Estudo observacional } \\
\text { transversal }\end{array}$ & $\begin{array}{l}\text { Avaliar a cobertura, o tempo de coleta e a incidência de doença } \\
\text { falciforme obtida no programa de TN da BA de } 2007-2009\end{array}$ & Gaz. méd. Bahia \\
\hline $\begin{array}{l}\text { COSTA SN, et al., } \\
2012 .\end{array}$ & $\begin{array}{l}\mathrm{PKU}, \mathrm{HC} \text { e } \\
\mathrm{HB}\end{array}$ & Estudo descritivo & $\begin{array}{l}\text { Avaliar a cobertura do PTN e incidência de doenças triadas em } \\
\text { Cruz das Almas e Valença, Bahia. }\end{array}$ & $\begin{array}{l}\text { Revista Baiana de } \\
\text { Saúde Pública }\end{array}$ \\
\hline $\begin{array}{l}\text { VESPOLI S, et al., } \\
2011 .\end{array}$ & $\begin{array}{l}\text { PKU, HB e } \\
\mathrm{HC}\end{array}$ & $\begin{array}{l}\text { Estudo transversal e } \\
\text { observacional }\end{array}$ & $\begin{array}{l}\text { Descrever e avaliar as prevalências das alterações detectadas pelo } \\
\text { PNTN no município de Araraquara durante o período de abril a } \\
\text { dezembro de } 2009 \text {, a fim de caracterizar o perfil populacional do } \\
\text { município quanto às doenças detectadas pelo programa. }\end{array}$ & $\begin{array}{l}\text { Rev Ciênc Farm } \\
\text { Básica Apl. }\end{array}$ \\
\hline
\end{tabular}

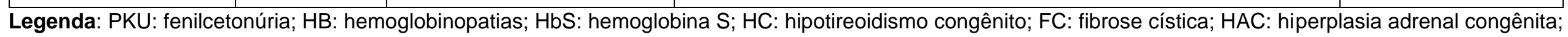
AF: anemia falciforme; DBT: deficiência de biotinidase; TN: triagem neonatal; RN: recém-nascidos; PNTN: Programa Nacional de Triagem Neonatal.

Fonte: Baggio FL et al., 2019. 
Quanto aos estados de realização dos estudos, identificou-se os seguintes estados: Mato Grosso do Sul, Paraná, Goiás, Santa Catarina, Piauí, Ceará, Rondônia, Bahia, Tocantins, Minas Gerais, São Paulo, Mato Grosso e Distrito Federal (Figura 1).

Figura 1 - Estados de realização dos estudos selecionados.

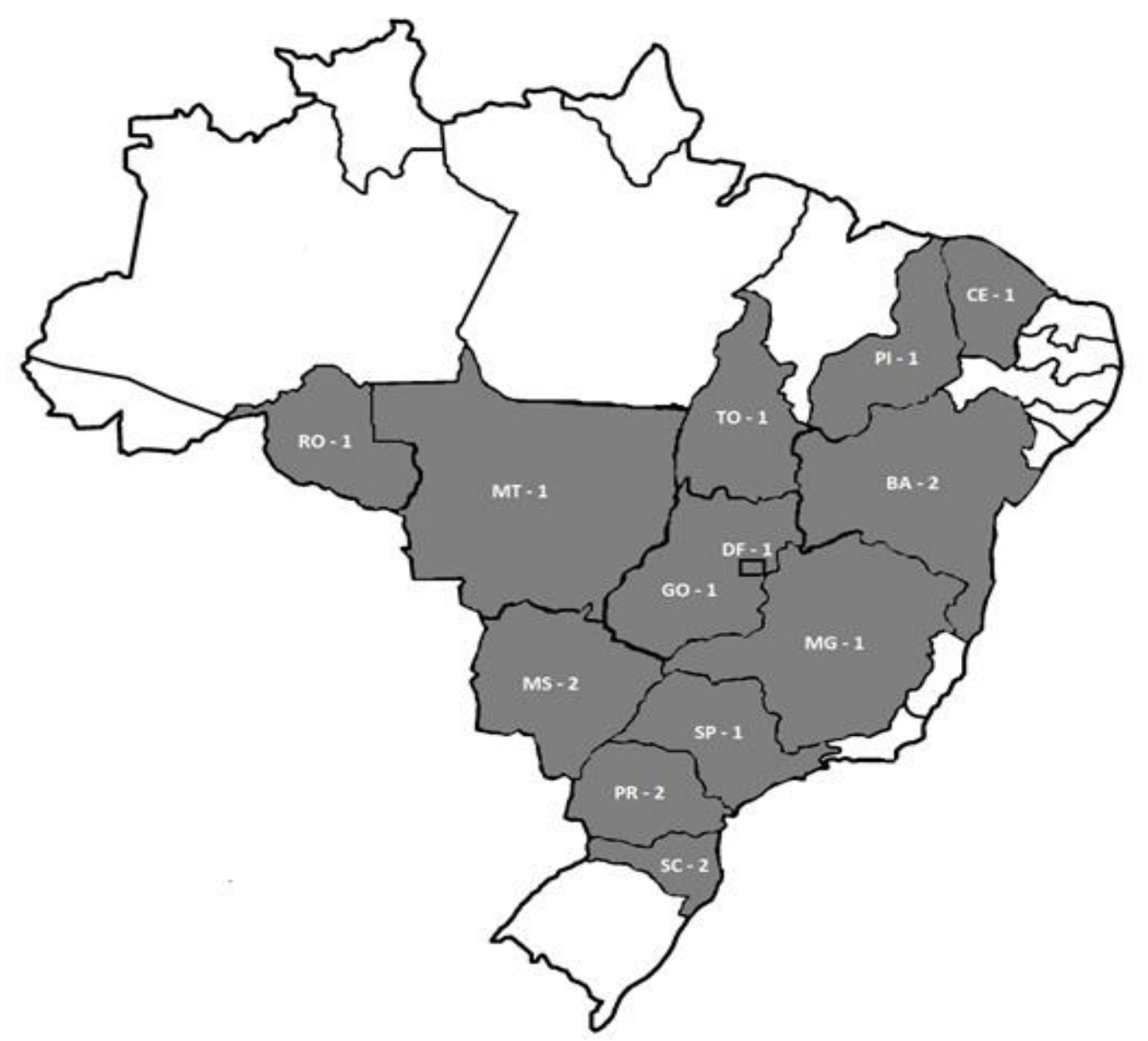

Fonte: Baggio FL et al., 2019.

A prevalência das doenças foi dividida para análise em: número total de nascidos vivos (NV) encontrados pelos respectivos autores, número total de casos, porcentagem de casos de acordo com a população analisada e prevalência representada em relação ao número de casos por nascidos vivos. Relativo às prevalências encontradas, foram atingidas diferenças consideráveis entre as populações analisadas devido à discrepância de amostra utilizada pelos autores (Tabela 2). 
Tabela 2 - Prevalência das doenças analisadas em cada estudo selecionado.

\begin{tabular}{|c|c|c|c|c|c|}
\hline & Doença & Total & Casos & $\%$ & Prevalência \\
\hline \multirow{2}{*}{$\begin{array}{l}\text { CARVALHO DCSN, } \\
\text { et al., } 2017 .\end{array}$} & PKU & 2893 & 2 & 0,069 & $1: 1447$ NV \\
\hline & $\mathrm{HC}$ & 2893 & 3 & 0,104 & 1:964 NV \\
\hline DINIZ D, et al., 2009. & AF & 116271 & 109 & 0,094 & 1:1067 NV \\
\hline \multirow{5}{*}{$\begin{array}{c}\text { NUNES AKC, et al., } \\
2013 .\end{array}$} & $\mathrm{HC}$ & 378337 & 132 & 0,035 & $1: 2876^{*} \mathrm{NV}$ \\
\hline & FC & 378337 & 76 & 0,020 & $1: 5121^{*} \mathrm{NV}$ \\
\hline & $\mathrm{HB}$ & 378337 & 24 & 0,006 & $1: 14446 * N V$ \\
\hline & $\mathrm{HAC}$ & 378337 & 77 & 0,020 & $1: 36838^{*} \mathrm{NV}$ \\
\hline & PKU & 378337 & 26 & 0,007 & $1: 28862^{*} \mathrm{NV}$ \\
\hline $\begin{array}{c}\text { SOUZA RAV, et al., } \\
2009 .\end{array}$ & $\mathrm{HB}$ & 13835 & 242 & 1,749 & $1: 57 \mathrm{NV}$ \\
\hline $\begin{array}{l}\text { LOPES FM, et al., } \\
2010 .\end{array}$ & PKU & 325308 & 11 & 0,003 & 1:29575 NV \\
\hline \multirow{5}{*}{ LUZ GS, et al., 2008.} & PKU & 20529 & 1 & 0,005 & 1:20529 NV \\
\hline & $\mathrm{HC}$ & 20529 & 9 & 0,044 & 1:2281 NV \\
\hline & $\mathrm{HB}$ & 20529 & 6 & 0,029 & 1:3421 NV \\
\hline & FC & 20529 & 2 & 0,010 & 1:10264 NV \\
\hline & DBT & 20529 & 3 & 0,015 & 1:6843 NV \\
\hline $\begin{array}{l}\text { HOLSBACH DR, et } \\
\text { al., } 2008 .\end{array}$ & $\mathrm{HB}$ & 190809 & 2624 & 1,375 & $1: 73 \mathrm{NV}$ \\
\hline $\begin{array}{l}\text { SIQUEIRA BR, et al., } \\
2009 .\end{array}$ & AF & 25446 & 9 & 0,035 & 1:2827 NV \\
\hline \multicolumn{6}{|l|}{ BALENSIEFER TK e } \\
\hline $\begin{array}{c}\text { YAMAGUCHI MU, } \\
2018 .\end{array}$ & HB & 1005 & 21 & 2,090 & $1: 48 \mathrm{NV}$ \\
\hline $\begin{array}{l}\text { REIS FMS, et al., } \\
2018 .\end{array}$ & HB & 69180 & 3747 & 5,416 & 1:18 NV \\
\hline $\begin{array}{c}\text { ELLER R e SILVA } \\
\text { DB, } 2016 .\end{array}$ & $\mathrm{AF}$ & 730412 & 39 & 0,005 & 1:18729 NV \\
\hline $\begin{array}{l}\text { BARRA CB, et al., } \\
2012 .\end{array}$ & HAC & 159415 & 8 & 0,005 & 1:19939 NV \\
\hline \multirow{2}{*}{$\begin{array}{l}\text { MENDES LC, et al., } \\
2013 .\end{array}$} & PKU & 254782 & 9 & 0,003 & 1:28309 NV \\
\hline & $\mathrm{HC}$ & 254782 & 55 & 0,021 & 1:4632 NV \\
\hline \multirow{2}{*}{$\begin{array}{c}\text { STRANIERI I e } \\
\text { TAKANO OA, } 2009 .\end{array}$} & PKU & 66337 & 2 & 0,003 & $1: 33068 \mathrm{NV}$ \\
\hline & $\mathrm{HC}$ & 66337 & 7 & 0,010 & $1: 9448$ NV \\
\hline $\begin{array}{l}\text { AMORIM T, et al., } \\
2010 .\end{array}$ & $\mathrm{AF}$ & 581060 & 966 & 0,166 & $1: 602$ NV \\
\hline \multirow{3}{*}{$\begin{array}{c}\text { COSTA SN, et al., } \\
2012 .\end{array}$} & PKU & 16896 & 0 & 0 & 0 \\
\hline & $\mathrm{HB}$ & 16896 & 34 & 0,201 & $1: 497 \mathrm{NV}$ \\
\hline & $\mathrm{HC}$ & 16896 & 8 & 0,047 & $1: 2112$ NV \\
\hline \multirow{3}{*}{$\begin{array}{l}\text { VESPOLI S, et al., } \\
2011 .\end{array}$} & $\mathrm{PKU}$ & 1749 & 1 & 0,057 & $1: 1749$ NV \\
\hline & $\mathrm{HC}$ & 1710 & 1 & 0,058 & $1: 1710 \mathrm{NV}$ \\
\hline & HB & 1583 & 45 & 2,842 & 1:35 NV \\
\hline
\end{tabular}

Legenda: *Prevalência analisada pelo autor de acordo com a média 2004 a 2008. PKU: fenilcetonúria; HC: hipotireoidismo congênito; AF: anemia falciforme; HB: hemoglobinopatias; HAC: hiperplasia adrenal congênita; FC: fibrose cística; DBT: deficiência de biotinidase; NV: nascidos vivos. Fonte: Baggio FL et al., 2019. 


\section{DISCUSSÃO}

A triagem neonatal no Brasil é pública, gratuita e obrigatória, sendo um direito de todas as crianças, diferente da maioria dos países no mundo, onde a saúde pública federal não abrange a todos, em número de triados e investimento financeiro. Em um comparativo por regiões do Brasil, observa-se heterogeneidade de estudos sobre as prevalências das doenças triadas (BOTLER J, et al., 2010; SOMMER CK, et al., 2006).

Os estudos sobre anemia falciforme e/ou outras hemoglobinopatias estão presentes em todas as regiões do Brasil (principalmente nas regiões Sul, Nordeste e Centro-Oeste, com três estudos cada uma), assim como os estudos sobre hipertireoidismo congênito, que também estão presentes em todas as regiões (sendo dois estudos na região Nordeste, um estudo na região Sudeste, dois estudos na região Sul, um estudo na região Centro-Oeste e um estudo na região Norte). As hemoglobinopatias (incluindo anemia falciforme) foram as doenças com as maiores prevalências encontradas pelos autores (ELLER R e SILVA DB, 2016).

Ao analisar a prevalência dos estudos selecionados, representados na Figura 2, a fenilcetonúria apresenta distribuição ampla, presente em todas as regiões do Brasil, sendo: região Nordeste dois estudos), região Sudeste (um estudo), região Sul (dois estudos), região Centro-Oeste (dois estudos) e região Norte (um estudo) (CARVALHO DCSN, et al., 2017; NUNES AKC, et al., 2013; LOPES FM, et al., 2010; LUZ GS, et al., 2008; MENDES LC, et al., 2013; STRANIERI I e TAKANO OA, 2009; COSTA SN, et al., 2012; VESPOLI S, et al., 2011).

Os estudos sobre a prevalência de hiperplasia adrenal congênita se restringiram à região Sul (um estudo no Paraná) e à região Sudeste (um estudo em Minas Gerais). A região Sul foi a única que produziu conhecimento acerca da prevalência da fibrose cística e da deficiência de biotinidase. Foram encontrados dois estudos sobre a prevalência de fibrose cística e apenas um estudo em relação à prevalência da deficiência de biotinidase (NUNES AKC, et al., 2013; LUZ GS, et al., 2008).

Em relação à quantidade de produção de conhecimento sobre a prevalência das doenças triadas pela TN no Brasil no período em questão (2008-2018), a região Centro-Oeste foi a que apresentou maior quantidade de estudos (cinco estudos), enquanto as regiões Norte e Sudeste apresentaram menor quantidade (dois estudos cada) (SIQUEIRA BR, et al., 2009; MENDES LC, et al., 2013; VESPOLI S, et al., 2011; BARRA CB, et al., 2012).

Quanto à quantidade de doenças analisadas pelos estudos, a região Sul foi a que apresentou análise mais ampla (entre seus quatro estudos, todas as doenças foram contempladas: AF, HB, HC, HAC, DBT, FC e PKU). Em contrapartida, a região Norte cobriu apenas três doenças (PKU, HC e AF) em seus estudos (SIQUEIRA BR, et al., 2009; MENDES LC, et al., 2013).

O grupo de doenças mais pesquisadas foi o das hemoglobinopatias (12 estudos, considerando também os estudos sobre anemia falciforme), podendo ser explicada pela diversidade étnica brasileira, principalmente pela elevada porcentagem da população sendo composta por afrodescendentes (BALENSIEFER TK e YAMAGUCHI MU, 2018). Todos os estudos encontrados da região Sul analisaram as hemoglobinopatias (em geral ou restritamente à forma de anemia falciforme). A doença menos pesquisada foi a deficiência de biotinidase, com apenas um estudo. Nenhum estudo de prevalência englobou os seis grupos de doenças detectadas pela TN. A representação das doenças que tiveram suas prevalências analisadas por estado do Brasil foi demonstrada a seguir (Figura 2). 
Figura 2 - Doenças cujas prevalências foram analisadas por estado

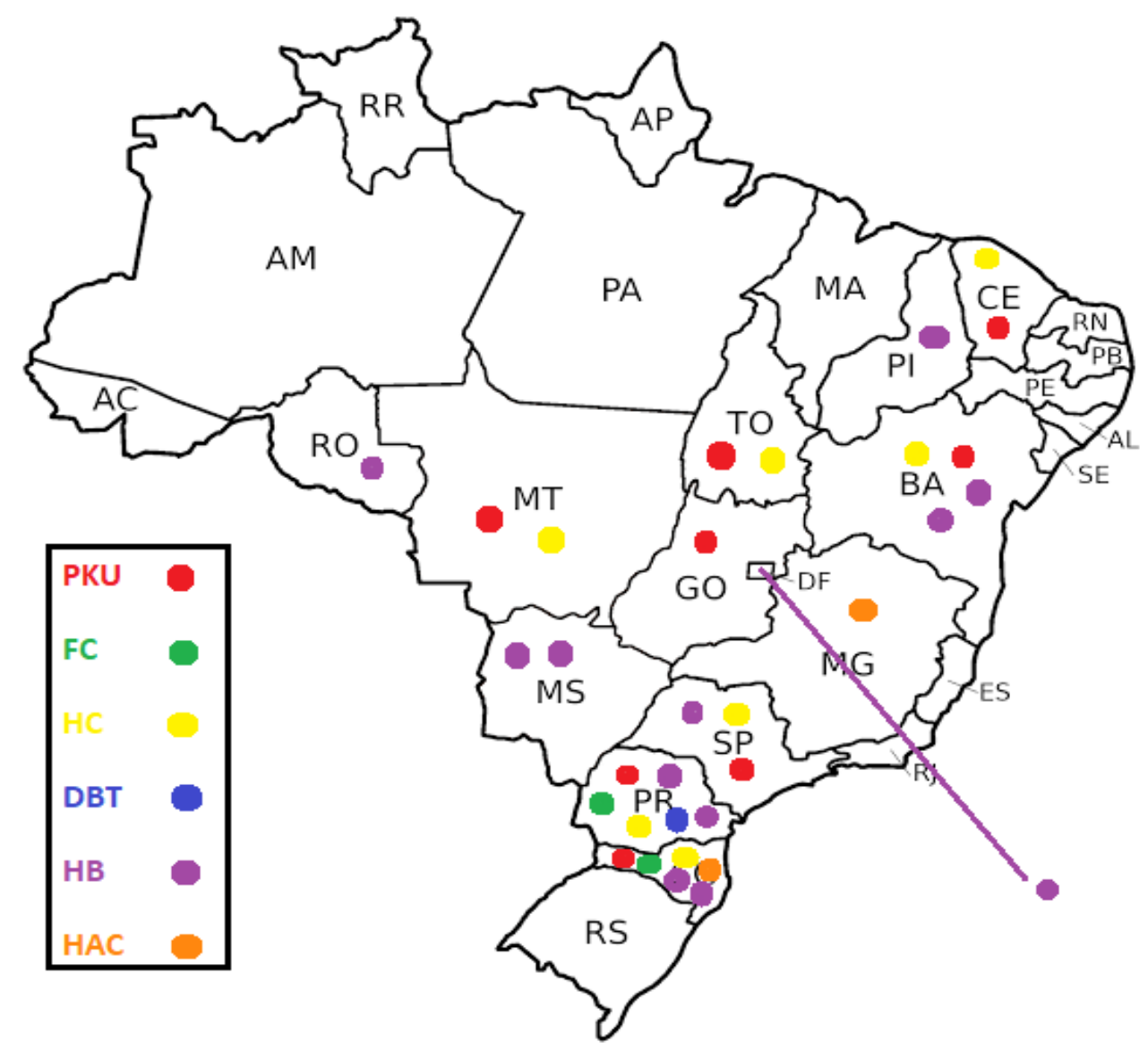

Fonte: Baggio FL et al., 2019.

Brasil (2018) afirma que o percentual de coleta do teste na idade ideal foi de 44,85\% em 2004 para 53,51\% em 2017, sendo o maior índice de coleta em idade ideal no ano de 2011, com 61,97\% dos recém-nascidos triados até o 5 dia de vida. Já o percentual de cobertura nacional do PNTN aumentou de 74,98\% em 2004 para $85,80 \%$ em 2017, sendo o maior índice registrado.

O cenário atual da TN no Brasil é discrepante em relação a outros países. Em um comparativo mundial, há uma variação de 100\% de cobertura em alguns países, como o Japão, e 1\% em outros, como a Índia. Therrell BL, et al. (2015) constata que a doença mais triada pela TN no mundo é o hipotireoidismo congênito, sendo a única doença triada em diversos países, como a Colômbia.

As doenças triadas pelos programas de triagem neonatal (PTN) conquistaram destaque após as alterações da configuração de morbimortalidade em relação ao desenvolvimento social, também marcada como mudança no curso da demografia e da epidemiologia. Dessa forma, ao retratar o cenário mundial, é válido ressaltar as divergências existentes, haja vista que a TN será moldada de acordo com a condição socioeconômica e cultural do país (THERRELL BL, et al., 2015).

Botler J, et al. (2010) afirma que os países da América do Norte (Estados Unidos e Canadá) possuem programas descentralizados em seus territórios. No que diz respeito aos estados norte-americanos, existem leis estaduais que tornam a TN obrigatória e responsabilidade de saúde pública.

As doenças triadas obrigatoriamente, em todo o território norte-americano, são: fenilcetonúria, hipotireoidismo congênito, hemoglobinopatias, hiperplasia adrenal congênita, deficiência de biotinidase, fibrose cística e galactosemia. Outras doenças têm triagem heterogênea entre os estados, sendo bastante prevalentes a imunodeficiência combinada grave (SCID), os distúrbios de ácidos graxos e distúrbios de aminoácidos. 
Nos Estados Unidos da América a coleta de testes de triagem neonatal varia na maneira de financiamento, sendo o programa não ligado estritamente à esfera federal. Por ser uma obrigatoriedade estatal, o governo custeia alguns exames para os cidadãos de famílias menos abastadas, além de ter participação inconstante em relação ao subsídio dos programas de triagem pelos 50 estados americanos.

Sobre o processo da TN nos Estados Unidos, relata-se que há recomendação para que seja realizado o teste no momento da alta hospitalar, no entanto, diversos programas espalhados pelo país recomendam, de modo não compulsório, a repetição de testes. Apenas nove estados norte-americanos decretaram a realização de uma segunda triagem de modo mandatório, a fim de aumentar a acurácia das avaliações, sendo feita preferencialmente entre a idade de 7 e 14 dias de vida.

Aproximadamente $25 \%$ dos RN são submetidos a uma segunda coleta (THERRELL BL e ADAMS J, 2007). No Brasil, somente o Paraná por meio da Fundação Ecumênica de Proteção ao Excepcional (FEPE) realiza a primeira coleta na maternidade, na alta hospitalar (FUNDAÇÃO ECUMÊNICA DE PROTEÇÃO AO EXCEPCIONAL, 2019). Os demais estados possibilitam a primeira coleta na Unidade Básica de Saúde ou na Secretaria de Saúde.

Bombard Y, et al. (2012) ressalta que no Canadá apenas três doenças são triadas em todos os programas provinciais de triagem: fenilcetonúria, hipotireoidismo congênito e deficiência de acil-CoA desidrogenase de cadeias médias (MCAD). Em 2010, iniciou-se uma iniciativa para a triagem de fibrose cística que foi acatada por quase todas as províncias. O governo federal não tem qualquer participação nos PTN, nem existe qualquer estratégia nacional, padrões ou diretrizes que orientem os seus desempenhos, assim com os Estados Unidos, sendo um programa descentralizado.

Já no que se refere à Europa, o desenvolvimento da TN foi lento e heterogêneo, sendo desenvolvido inicialmente nos países ocidentais e posteriormente no Leste Europeu. Na maior parte dos países ocidentais europeus, existe a meta de constatar indivíduos de risco para omissão de coleta, sendo estes em geral integrantes de populações marginalizadas da sociedade europeia, como crianças migrantes ou imigrantes de países subdesenvolvidos. Todos os países europeus possuem programas de triagem, exceto a Albânia (HOLMES D, 2012).

$\mathrm{Na}$ América Latina, a triagem neonatal ocorre há mais de 20 anos em países como Cuba, Costa Rica, Uruguai e Chile. A cobertura de RN nesses países chega a 99\% (THERRELL BL, et al., 2015). Em países como Chile, México, Argentina, Colômbia e Venezuela, é possível fazer o teste ampliado na rede privada, triando mais doenças do que o teste gratuito governamental.

O programa da TN no Chile apresentou um projeto piloto em 2015 de expansão para triagem de 25 doenças. Na Argentina, os programas de triagem foram consolidados na década de 1990, e as triagens englobam hipotireoidismo congênito, fenilcetonúria, galactosemia, deficiência de biotinidase, hiperplasia adrenal congênita e distúrbios de oxidação de ácidos graxos.

A cobertura da triagem na Argentina é de aproximadamente $90 \%$. A TN no Paraguai é relativamente recente, sendo o projeto iniciado em 2003. O Paraguai tria 3 doenças (hipotireoidismo congênito, fenilcetonúria e fibrose cística) com cobertura de apenas 60\% em 2013 em instituições particulares e $94 \%$ de cobertura em instituições públicas (BORRAJO GJC, 2012).

Alguns países possuem situação de TN crítica. A Bolívia tornou obrigatória a triagem apenas para hipotireoidismo congênito, mas não implementou um programa nacional para tal. A Guatemala tria hipotireoidismo congênito, fenilcetonúria e hiperplasia adrenal congênita, porém com cobertura de apenas $1 \%$ dos RN. Em El Salvador, o programa de triagem neonatal iniciou-se em 2008, mas foi descontinuado por falta de recursos. Já no Haiti, não há programas de triagem neonatal (THERRELL BL, et al., 2015).

A Coreia do Sul e o Japão apresentam taxas de cobertura de triagem de 99\% e 100\%, respectivamente, e ambos os países triam uma ampla gama de doenças: hipotireoidismo congênito, fenilcetonúria, galactosemia, doença de urina de xarope de bordo, homocistinúria, hiperplasia adrenal congênita, distúrbios de aminoácidos, e distúrbios de ácidos graxos. Em contrapartida, Paquistão e a Indonésia possuem apenas programas privados de triagem neonatal, com coberturas inferiores a $1 \%$ e $2 \%$ dos RN, respectivamente. Já 
Bangladesh possui triagem pública apenas para hipotireoidismo congênito, com uma cobertura inferior a 5\% dos NV (PADILLA CD e THERRELL BL, 2007).

Alguns obstáculos parecem interferir nas taxas de triagem neonatal insatisfatórias, tais como: economia pobre, educação em saúde insuficiente, falta de apoio governamental, alta hospitalar precoce e grande número de nascimentos fora do hospital (THERRELL BL, et al., 2015). Alguns itens foram identificados como essenciais para garantir programas de triagem efetivos: priorização e financiamento (total ou parcial) por parte do governo, educação e aceitação pública e participação governamental em programas de institucionalização.

Os dados obtidos mostram evidências científicas geográficas capazes de contextualizar e justificar a implantação de políticas públicas para doenças mais prevalentes. O Brasil encontra-se à frente de muitos países ao redor do mundo em relação à situação da TN, no entanto, muito ainda deve ser feito para atingir os indicadores de triagem de alguns países desenvolvidos, como o Japão.

\section{CONSIDERAÇÕES FINAIS}

O presente estudo evidencia a necessidade de ampliar as pesquisas sobre a prevalência das doenças triadas pela Triagem Neonatal no Brasil. Observa-se discrepância da produção de conhecimentos sobre prevalência das doenças entre os estados brasileiros. Constataram-se estudos que avaliaram maior número de doenças na região Sul, enquanto a região Norte apresenta escassez de pesquisas. É necessário ampliar a cobertura nacional da TN, assim como a produção de conhecimento, para possibilitar melhor delineamento de políticas públicas para amparo da população-alvo. Além disso, é fundamental aumentar o interesse científico por doenças pouco abordadas, como a deficiência de biotinidase, para fornecer dados que auxiliem na construção de uma atenção integral às crianças com doenças genéticas e suas famílias.

\section{REFERÊNCIAS}

1. AMORIM T, et al. Avaliação do programa de triagem neonatal da Bahia entre 2007 e 2009 - as lições da doença falciforme. Gazeta Médica da Bahia, 2010; 80:3(Ago-Out)10-13.

2. BALENSIEFER TK, YAMAGUCHI MU. Triagem neonatal de hemoglobinopatias em Maringá - PR. Revista Brasileira de Análises Clínicas, 2018; 50(2 supl.2):S8-13.

3. BARRA CB, et al. Triagem neonatal para hiperplasia adrenal congênita. Rev Assoc Med Bras, 2012; 58(4):459-464.

4. BENEFIELD LE. Implementing evidence-based practice in home care. Home Healtc Nurse, 2003; 21 (12): 804-809.

5. BOMBARD Y, et al. Citizens' values regarding research with stored samples from newborn screening in Canada. Pediatrics. Pediatrics, 2012; 129(2):239-247.

6. BONFIM IM, et al. Perfil dos pacientes com fibrose cística atendidos no centro de referência pediátrico do Espírito Santo. Rev. Bras. Pesq. Saúde, Vitória, 2019; 21(1): 80-85, jan-mar.

7. BORRAJO GJC. Panorama epidemiológico de la fenilcetonuria (PKU) en Latinoamérica. Acta Pediátrica de México, 2012; 33(6):279-287.

8. BOTLER J, et al. Triagem neonatal - o desafio de uma cobertura universal e efetiva. Ciência \& Saúde Coletiva, 2010; 15(2):493-508.

9. BRASIL. 2018. In: Ministério da Saúde. Programa Nacional de Triagem Neonatal: Indicadores de Triagem Neonatal. Disponível em: http://www.saude.gov.br/acoes-e-programas/programa-nacional-da-triagem-neonatal/indicadores-datriagem-neonatal-no-brasil. Acesso em: 22 jul. 2019.

10. BRASIL. Ministério da Saúde. Secretaria de Assistência à Saúde/Coordenação-Geral de Atenção Especializada Manual de Normas, Técnicas e Rotinas Operacionais do Programa Nacional de Triagem Neonatal. Brasília-DF. 2002.

11. BRASIL. Ministério da Saúde. Secretaria de Atenção a Saúde. Departamento de Atenção Especializada e Temática. Triagem neonatal biológica: manual técnico / Ministério da Saúde, Secretaria de Atenção a Saúde, Departamento de Atenção Especializada e Temática. - Brasília: Ministério da Saúde, 2016.

12. CARVALHO DCSN, et al. Evolução do Programa de Triagem Neonatal em hospital de referência no Ceará: 11 anos de observação. ABCS Health Sci, 2017; 42(3);143-146.

13. COSTA SN, et al. Triagem neonatal para fenilcetonúria, hipotireoidismo congênito e hemoglobinopatias no Recôncavo Baiano: avaliação da cobertura em Cruz das Almas e Valença, Bahia, Brasil. Revista Baiana de Saúde Pública, 2012; 36(1):831-843.

14. DINIZ D, et al. Prevalência do traço e da anemia falciforme em recém-nascidos do Distrito Federal, Brasil, 2004 a 2006. Cad. Saúde Pública, 2009; 25(1):188-194.

15. ELLER R, SILVA DB. Evaluation of a neonatal screening program for sickle-cell disease. J. Pediatr, 2016; 92: 409413.

16. FUNDAÇÃO ECUMÊNICA DE PROTEÇÃO AO EXCEPCIONAL. 2019. In: Teste do pezinho. Disponível em: https://www.fepe.org.br/teste-do-pezinho/. Acesso em: 20 set. 2019. 
17. HOLMES D. Europe plays catch-up on neonatal screening as US skips ahead. Nature Medicine, 2012; 18(11):15961596.

18. HOLSBACH DR, et al. Ocorrência de hemoglobina S no estado de Mato Grosso do Sul, Brasil. J Bras Patol Med Lab, 2008; 44(4):277-282.

19. KOPACEK C, et al. Clinical and molecular profile of newborns with confirmed or suspicious congenital adrenal hyperplasia detected after a public screening program implementation. J Pediatr, 2019; 95(3):282-290.

20. LEÃO LL, AGUIAR MJB. Triagem neonatal: o que os pediatras deveriam saber. J Pediatr, 2008; 84(4):S80-90.

21. LERVOLINO LG, et al. Prevalence of sickle cell disease and sickle cell trait in national neonatal screening studies. Rev Bras Hematol Hemoter, 2011; 33(1):49-54.

22. LOPES FM, et al. Levantamento epidemiológico da fenilcetonúria no Estado de Goiás. Ensaios e Ciência: Ciências Biológicas, Agrárias e da Saúde, 2010; 14(2):61-70.

23. LUZ GS, et al. Prevalência das doenças diagnosticadas pelo Programa de Triagem Neonatal em Maringá, Paraná, Brasil: 2001-2006. Rev Gaúcha Enferm, 2008; 29(3):446-453.

24. MENDES KDS, et al. Revisão integrativa: método de pesquisa para a incorporação de evidências na saúde e na enfermagem. Texto Contexto Enferm, 2008; 17(4):758-764.

25. MENDES LC, et al. Evolução do programa de triagem neonatal no estado do Tocantins. Arq Bras Endocrinol Metab, 2013; 57(2):112-119.

26. NUNES AKC, et al. Prevalência de patologias detectadas pela triagem neonatal em Santa Catarina. Arq Bras Endocrinol Metab, 2013; 57(5):360-367.

27. PADILLA CD, THERRELL BL. Newborn screening in the Asia Pacific region. Journal of Inherited Metabolic Disease, 2007; 30(4): 490-506.

28. REIS FMS, et al. Incidência de hemoglobinas variantes em neonatos assistidos por um laboratório de saúde pública. Einstein (São Paulo), 2018; 16(2):1-7.

29. SIQUEIRA BR, et al. Incidência de anemia falciforme, traço falcêmico e perfil hemoglobínico dos casos diagnosticado na triagem neonatal no estado de Rondônia no ano de 2003. Saber Científico, 2009; 2(1):43-53.

30. SOCIEDADE BRASILEIRA DE PEDIATRIA. 2019. In: Teste do Pezinho ampliado deve ser oferecido no SUS. Disponível em: https://www.sbp.com.br/imprensa/detalhe/nid/teste-do-pezinho-ampliado-deve-ser-oferecido-no-susafirma-presidente-de-dc/. Acesso em: 06 jun. 2019.

31. SOMMER CK, et al. Triagem neonatal para hemoglobinopatias: experiência de um ano na rede de saúde pública do Rio Grande do Sul, Brasil. Cad Saúde Pública, 2006; 22(8):1709-1714.

32. SOUZA AS, et al. Etiologia do hipotireoidismo congênito e sua distribuição nas macrorregiões do estado de Minas Gerais. Revista Interdisciplinar Ciências Médicas, 2018; 2(2):22-28.

33. SOUZA RAV, et al. Programa de triagem neonatal para hemoglobinopatias em Dourados, MS - uma análise. Rev Bras Hematol Hemoter, 2010; 32(2):126-130.

34. STRANIERI I, TAKANO OA. Avaliação do Serviço de Referência em Triagem Neonatal para hipotireoidismo congênito e fenilcetonúria no Estado de Mato Grosso, Brasil. Arq Bras Endocrinol Metab, 2009; 53(4): 446-452.

35. THERRELL BL, ADAMS J. Newborn screening in North America. J Inherit Metab Dis, 2007; 30(4):447-465.

36. THERRELL BL, et al. Current status of newborn screening worldwide: 2015. Semin, 2015; 39(3):171-187.

37. VESPOLIS, et al. Análise das prevalências de doenças detectadas pelo programa nacional de triagem neonatal no município de Araraquara no ano de 2009. Rev Ciênc Farm Básica Apl, 2011; 32(2):269-273.

38. WITCHEL SF. Congenital Adrenal Hyperplasia. Journal of Pediatric \& Adolescent Gynecology, 2017; 24(3):116-126. 